\title{
AN APPROXIMATION TO DISCRETE OPTIMAL FEEDBACK CONTROLS
}

\author{
JINGHAO ZHU and ZHIQIANG ZOU
}

Received 3 November 2002

\begin{abstract}
We study discrete solutions of nonlinear optimal control problems. By value functions, we construct difference equations to approximate the optimal control on each interval of "small" time. We aim to find a discrete optimal feedback control. An algorithm is proposed for computing the solution of the optimal control problem.
\end{abstract}

2000 Mathematics Subject Classification: 49M25, 49K15.

1. Introduction and statement of the problem. For nonlinear analytic systems, there have been many works on control problems since the eighties of the last century. In [3, 4, 5, 6], Lie series was used in studying the controllability of nonlinear analytic systems. In [1], the discrete method in solving HamiltonJacobi-Bellman equations for value functions of nonlinear problems was discussed. In this paper, we use Lie series to construct difference equations by value functions in obtaining the discrete solutions of nonlinear optimal control problems. Taking advantage of the uniform convergence of Lie series on an interval of "small" time, we focus on the integral of the optimal control function. We aim to find a discrete optimal feedback control. We see that the optimal controls of a given problem can be constructed by these integral dates. We propose an algorithm which includes the process of pre-estimation and correction of an approximation to the solution of the optimal control problem.

We begin by considering the following nonlinear control system:

$$
\dot{x}=f(x)+G(x) u, \quad x(0)=x_{0} \in X \subset \mathbb{R}^{n}, \quad t \in[0, T],
$$

where $f: \mathbb{R}^{n} \rightarrow \mathbb{R}^{n}$ and $G: \mathbb{R}^{n} \rightarrow \mathbb{R}^{n \times m}$,

$$
G=\left(g_{1}, \ldots, g_{m}\right),
$$

are real analytic mappings. We consider the admissible controls $u(t)$, which take values in some compact set $U \subset \mathbb{R}^{m}$, to be integrable. Throughout this paper, it is assumed that the state space $X$ is bounded. Let $Q(x)$ be a Lipschitz function. Denote by $x_{u}(t)$ the solution of system (1.1) relative to the control $u$. We pose the following optimal control problem: find an admissible control 
$\hat{u}(\cdot)$ such that

$$
Q(\hat{x}(T))=\min _{u \in U}\left\{Q\left(x_{u}(T)\right)\right\}
$$

where $\hat{x}(\cdot)$ is the solution of (1.1) relative to the optimal control $\hat{u}(\cdot)$.

2. A lemma on Lie series. We introduce Lie series for system (1.1) and real analytic function $P(x)$ (see [4, pages 698-699]) in the following lemma.

LEMMA 2.1. Let $P(x)$ be a real analytic function on $\mathbb{R}^{n}$ and let $\mathscr{K}$ be compact in $\mathbb{R}^{n}$. Suppose that $x_{0} \in \mathscr{K}$ and consider the admissible controls $u(\cdot)$ satisfying $\|u(\cdot)\| \leq M$, a.e. on $[0, T]$. The solution of (1.1) corresponding to an admissible control $u(\cdot)$ is denoted by $x_{u}(\cdot)$. For a given positive integer $l$, denote $N=$ $m l$. Further, define $f_{0}=f$ and $f_{i}=g_{i}, i=1,2, \ldots, m$. Meanwhile, for each positive integer $k$, denote $I_{k}=\left(i_{1}, i_{2}, \ldots, i_{k}\right), 0 \leq i_{j} \leq m(j=1,2, \ldots, k)$, and $\left|I_{k}\right|=i_{1}+i_{2}+\cdots+i_{k}$. Further set

$$
\begin{aligned}
\operatorname{Ser}_{N}(u)(P)\left(t, x_{0}\right) & \\
= & \sum_{i=0}^{m}\left\{\int_{0}^{t} u_{i}(s) d s\right\}\left(f_{i} P\right)\left(x_{0}\right) \\
& +\sum_{i+j \leq 2 m}\left\{\int_{0}^{t} \int_{0}^{s} u_{i}(s) u_{j}(\tau) d \tau d s\right\}\left(f_{i} f_{j} P\right)\left(x_{0}\right)+\cdots \\
& +\sum_{\left|I_{l}\right| \leq N}\left\{\int_{0}^{t} \int_{0}^{\tau_{l}} \cdots \int_{0}^{\tau_{2}} u_{i_{l}}\left(\tau_{l}\right) \cdots u_{i_{1}}\left(\tau_{1}\right) d \tau_{1} \cdots d \tau_{l}\right\}\left(f_{i_{l}} \cdots f_{i_{1}} P\right)\left(x_{0}\right),
\end{aligned}
$$

where $u_{0}(t) \equiv 1$ and $\left(f_{i_{1}} P\right)(x)=L_{f_{i_{1}}} P$, and in turn, for each positive integer $k,\left(f_{i_{k}} \cdots f_{i_{1}} P\right)(x)=\left(f_{i_{k}} \cdots f_{i_{2}}\left(f_{i_{1}} P\right)\right)(x)$. Then there exists a positive number $\delta<1$ which depends only on $P, f, G, M$, and $\mathscr{K}$ such that if $0<t<\delta$,

$$
P\left(x_{u}(t)\right)-P\left(x_{0}\right)=\operatorname{Ser}_{N}(u)(P)\left(t, x_{0}\right)+R_{N}(u)(P)\left(t, x_{0}\right),
$$

where $R_{N}(u)(P)\left(t, x_{0}\right)$ is uniformly convergent to zero, when $N \rightarrow \infty$, as long as $x_{0} \in \mathscr{K}$ and $\|u(s)\| \leq M$, a.e. on $[0, t]$. Moreover, for the sufficiently large $N$,

$$
\begin{gathered}
\left|R_{N}(u)(P)\left(t, x_{0}\right)\right|<2 t^{2}, \\
\left|\operatorname{Ser}_{N}(u)(P)\left(t, x_{0}\right)-\sum_{i=0}^{m}\left\{\int_{0}^{t} u_{i}(s) d s\right\}\left(f_{i} P\right)\left(x_{0}\right)\right|<\tilde{C} t^{2},
\end{gathered}
$$

where $\tilde{C}$ only depends on $N, \mathscr{K}, M, m, f_{i}(i=0,1,2, \ldots, m)$, and $P$.

Proof. Since $P(x), f_{i}(x)$ are real analytic and $\mathscr{K}$ is compact, [4, Proposition 4.3] indicates that there is a positive $\delta<1$ such that, when $0<t<\delta$, $R_{N}(u)(P)\left(t, x_{0}\right)$ is uniformly convergent to zero for all admissible controls 
$u(\cdot)$ (satisfying $\|u(\cdot)\| \leq M$, a.e. on $[0, T]$ ) and all $x_{0}$ in $\mathscr{K}$. Following the proof of Proposition 4.3 due to Sussmann [4, pages 698-699], we can get a constant $C>0$ only depending on $N, \mathscr{K}, M, m, f_{i}(i=0,1,2, \ldots, m)$, and $P$ such that, when $0<t<\delta<1$,

$$
\begin{gathered}
\left|R_{N}(u)(P)\left(t, x_{0}\right)\right| \leq[\operatorname{CMt}(m+1)]^{N+1}, \\
\operatorname{CMt}(m+1)<\frac{1}{2} .
\end{gathered}
$$

It is easy to see from (2.6) that, when $N$ is sufficiently large, we have

$$
C M t^{(N-1) /(N+1)}(m+1)<1 .
$$

Consequently, by (2.5) and (2.7), we conclude (2.3) by the following estimation:

$$
\begin{aligned}
\left|R_{N}(u)(P)\left(t, x_{0}\right)\right| & \leq C^{N+1} M^{N+1} t^{N-1}(m+1)^{N+1} t^{2} \\
& \leq\left[C M t^{(N-1) /(N+1)}(m+1)\right]^{N+1} t^{2} \leq 2 t^{2} .
\end{aligned}
$$

On the other hand, fixing the positive $N=l m$, by (2.1), we see that, by (2.2),

$$
\begin{aligned}
\mid & \operatorname{Ser}_{N}(u)(P)\left(x_{0}\right)-\sum_{i=0}^{m}\left\{\int_{0}^{t} u_{i}(s) d s\right\}\left(f_{i} P\right)\left(x_{0}\right) \mid \\
\leq & \sum_{i+j \leq 2 m}\left\{\int_{0}^{t} \int_{0}^{s}\left|u_{i}(s)\right|\left|u_{j}(\tau)\right| d \tau d s\right\}\left|\left(f_{i} f_{j} P\right)\left(x_{0}\right)\right|+\cdots \\
& +\sum_{\left|I_{l}\right| \leq N}\left\{\int_{0}^{t} \int_{0}^{\tau_{l}} \cdots \int_{0}^{\tau_{2}}\left|u_{i_{l}}\left(\tau_{l}\right)\right| \cdots\left|u_{i_{1}}\left(\tau_{1}\right)\right| d \tau_{1} \cdots d \tau_{l}\right\}\left|\left(f_{i_{l}} \cdots f_{i_{1}} P\right)\left(x_{0}\right)\right| \\
\leq & B_{N, \mathscr{K}}\left(M^{2} t^{2}+\cdots+M^{l+1} t^{l+1}\right) \\
\leq & B_{N, \mathscr{K}}\left(M^{2}+M^{3} t+\cdots+M^{l+1} t^{l-1}\right) t^{2},
\end{aligned}
$$

where $B_{N, \mathscr{K}}$ only depends on $N, \mathscr{K}, f_{i}(i=0,1,2, \ldots)$, and $P$. Since $t<\delta<1$, from (2.9), we conclude (2.4).

3. The formulation of discrete solutions to the optimal control problem. We define the value function, for $(t, x) \in[0, T] \times X$,

$$
V(t, x):=\inf _{u \in U}\left\{Q\left(x_{u}(T, x)\right)-Q(x)\right\},
$$

where $x_{u}(s, x)$ is the solution of the following equation:

$$
\dot{x}=f(x)+G(x) u(s), \quad x(t)=x, \quad s \in[t, T] .
$$

It is well known that the value function is granted the following boundary condition:

$$
V(T, x)=0 \quad \forall x \in X
$$


We introduce the discrete scheme as follows. For a given positive integer $L$, divide $[0, T]$ into $L$ parts: $[(i-1) T / L, i T / L], i=1,2, \ldots, L$. Denote $t_{i}=i T / L$. Let $x_{i}, i=1,2, \ldots, L$, be the state point associated with $t_{i}$. For every $j, j=1,2, \ldots, n$, denote $e_{j}^{T}=(0, \ldots, 0,1,0, \ldots, 0)$ which has a 1 on the $j$ th place and zeros on other places. For every vector $x \in \mathbb{R}^{n}, x^{(j)}=e_{j}^{T} x$, which is denoted by $P_{j}(x)$. We need the following elementary lemma.

LEMMA 3.1. Let $\left\{x_{i}, i=1, \ldots, L\right\}$ be a set of state points. Suppose that, for each $i \in\{1,2, \ldots, L\}$,

$$
V\left(t_{i}, x_{i}\right)=V\left(0, x_{0}\right)+Q\left(x_{0}\right)-Q\left(x_{i}\right) .
$$

If there exists an admissible control $\hat{u}_{i}(\cdot)$ in $\left[t_{i-1}, t_{i}\right]$ steering system (1.1) from $x_{i-1}$ to $x_{i}, i=1,2, \ldots, L$, then the admissible control $\hat{u}(\cdot)$ which equals $u_{i}(\cdot)$ on each $\left[t_{i-1}, t_{i}\right]$ is an optimal control for the nonlinear optimal control problem (1.3).

Proof. By the formulation of $\hat{u}(\cdot)$, we see that

$$
x_{L}=x_{\hat{u}}(T)
$$

By (3.3) and (3.4), we have, for $i=L$,

$$
0=V\left(T, x_{L}\right)=V\left(0, x_{0}\right)+Q\left(x_{0}\right)-Q\left(x_{L}\right)
$$

Thus

$$
V\left(0, x_{0}\right)=Q\left(x_{\hat{u}}(T)\right)-Q\left(x_{0}\right)
$$

But (3.7) means that $\hat{u}(\cdot)$ is an optimal control of (1.3) according to definition (3.1).

REMARK 3.2. By Lemma 2.1, we see that when $\tau(=T / L)$ is sufficiently small, $\hat{u}(\cdot)$, given by Lemma 3.1, steers $x_{i-1}$ to $x_{i}$, which amounts to, for $j=1,2, \ldots, n$,

$$
\begin{aligned}
x_{i}^{(j)}-x_{i-1}^{(j)} & =\operatorname{Ser}_{N}(\hat{u})\left(P_{j}\right)\left(\tau, x_{i-1}\right)+R_{N}(\hat{u})\left(P_{j}\right)\left(\tau, x_{i-1}\right) \\
& =\tau\left(f P_{j}\right)\left(x_{i-1}\right)+\sum_{k=1}^{m}\left\{\int_{t_{i-1}}^{t_{i}} \hat{u}_{i}^{(k)}(s) d s\right\}\left(g_{k} P_{j}\right)\left(x_{i-1}\right)+O\left(\tau^{2}\right),
\end{aligned}
$$

where the term $R_{N}(\hat{u})\left(P_{j}\right)\left(\tau, x_{i-1}\right)$ converges uniformly to zero $(N \rightarrow \infty)$. On the other hand, condition (3.4) ensures the optimality of state points.

Now we derive the following difference equations. For each $i=0,1,2, \ldots, L-1$, when $x_{i}$ is obtained,

$$
x_{i+1}^{(j)}-x_{i}^{(j)}=\tau e_{j}^{T} f\left(x_{i}\right)+\sum_{k=1}^{m} S_{k}(i) e_{j}^{T} g_{k}\left(x_{i}\right), \quad j=1,2, \ldots, n,
$$


where for $i=0,1, \ldots, L-1, S_{k}(i)$ stands for the following integral form for an admissible control $u(\cdot)$ :

$$
S_{k}(i)=\int_{t_{i}}^{t_{i+1}} u^{(k)}(s) d s
$$

We establish

$$
V\left(t_{i+1}, x_{i+1}\right)=V\left(0, x_{0}\right)+Q\left(x_{0}\right)-Q\left(x_{i+1}\right) .
$$

We see that (3.9) can be rewritten in the vector form as follows:

$$
x_{i+1}=x_{i}+\tau f\left(x_{i}\right)+\sum_{k=1}^{m} S_{k}(i) g_{k}\left(x_{i}\right) .
$$

Substituting expression (3.12) for $x_{i+1}$ in (3.11), we have

$$
\begin{aligned}
& V\left[t_{i+1}, x_{i}+\tau f\left(x_{i}\right)+\sum_{k=1}^{m} S_{k}(i) g_{k}\left(x_{i}\right)\right] \\
& =V\left(0, x_{0}\right)+Q\left(x_{0}\right)-Q\left[x_{i}+\tau f\left(x_{i}\right)+\sum_{k=1}^{m} S_{k}(i) g_{k}\left(x_{i}\right)\right] .
\end{aligned}
$$

The discrete solution for each $i$ will be constructed by solving (3.13) for

$$
S_{1}(i), S_{2}(i), \ldots, S_{m}(i)
$$

satisfying

$$
\left|S_{k}(i)\right| \leq \frac{T \sup _{u \in U}\|u\|}{L}, \quad k=1,2, \ldots, m .
$$

Next suppose we have got $x_{i+1}$ such that

$$
V\left(t_{i+1}, x_{i+1}\right)=V\left(0, x_{0}\right)+Q\left(x_{0}\right)-Q\left(x_{i+1}\right),
$$

and $\tilde{S}_{k}(i),\left|\tilde{S}_{k}(i)\right| \leq T \sup _{u \in U}\|u\| / L(k=1,2, \ldots, m)$, such that

$$
x_{i+1}=x_{i}+\tau f\left(x_{i}\right)+\sum_{k=1}^{m} \tilde{S}_{k}(i) g_{k}\left(x_{i}\right) .
$$

We take a control $\tilde{u}_{i}(\cdot)$ on each $\left[t_{i}, t_{i+1}\right], i=0,1, \ldots, L-1$, such that

$$
\tilde{S}_{k}(i)=\int_{t_{i}}^{t_{i+1}} \tilde{u}_{i}^{(k)}(s) d s .
$$

Suppose that $\tilde{u}_{i}(\cdot)$ steers system (1.1) from $x_{i}$ to $\tilde{x}_{i+1}$. We have, by Lemma 2.1,

$$
\tilde{x}_{i+1}=x_{i}+\tau f\left(x_{i}\right)+\sum_{k=1}^{m} \tilde{S}_{k}(i) g_{k}\left(x_{i}\right)+O\left(\tau^{2}\right) .
$$


By (3.17) and (3.19), we have

$$
x_{i+1}-\tilde{x}_{i+1}=O\left(\tau^{2}\right), \quad Q\left(x_{i+1}\right)-Q\left(\tilde{x}_{i+1}\right)=O\left(\tau^{2}\right) .
$$

Further, we have

$$
V\left(t_{i+1}, x_{i+1}\right)-V\left(t_{i+1}, \tilde{x}_{i+1}\right)=O\left(\tau^{2}\right)
$$

noting by hypothesis that $Q(x)$ is a Lipschitz function and so is $V(t, x)$ by Lemma 3.3. Therefore, we have $\tilde{S}_{k}(i),\left|\tilde{S}_{k}(i)\right| \leq T \sup _{u \in U}\|u\| / L(k=1,2, \ldots, m)$, such that

$$
V\left(t_{i+1}, \tilde{x}_{i+1}\right)=V\left(0, x_{0}\right)+Q\left(x_{0}\right)-Q\left(\tilde{x}_{i+1}\right)+O\left(\tau^{2}\right)
$$

Then define $x_{i+1}=\tilde{x}_{i+1}$ to carry out the process (3.11), (3.12), (3.16), (3.17), (3.18), and (3.19) again.

LEMMA 3.3. For a given $t, V(t, x)$ is a Lipschitz function with respect to $x$.

Proof. Given $x^{\prime}, x^{\prime \prime} \in X$, we show, for arbitrarily given $\epsilon>0$, that

$$
\left|V\left(t, x^{\prime}\right)-V\left(t, x^{\prime \prime}\right)\right| \leq C\left|x^{\prime}-x^{\prime \prime}\right|+\epsilon
$$

By definition (3.1), we are able to get admissible controls $u^{\prime}, u^{\prime \prime}$ such that

$$
\begin{gathered}
V\left(t, x^{\prime}\right)>Q\left(x_{u^{\prime}}\left(T, x^{\prime}\right)\right)-Q\left(x^{\prime}\right)-\epsilon, \\
V\left(t, x^{\prime \prime}\right)>Q\left(x_{u^{\prime \prime}}\left(T, x^{\prime \prime}\right)\right)-Q\left(x^{\prime \prime}\right)-\epsilon .
\end{gathered}
$$

Then we see that

$$
\begin{aligned}
& V\left(t, x^{\prime}\right)-V\left(t, x^{\prime \prime}\right) \leq Q\left(x_{u^{\prime \prime}}\left(T, x^{\prime}\right)\right)-Q\left(x^{\prime}\right)-\left[Q\left(x_{u^{\prime \prime}}\left(T, x^{\prime \prime}\right)\right)-Q\left(x^{\prime \prime}\right)-\epsilon\right], \\
& V\left(t, x^{\prime \prime}\right)-V\left(t, x^{\prime}\right) \leq Q\left(x_{u^{\prime}}\left(T, x^{\prime \prime}\right)\right)-Q\left(x^{\prime \prime}\right)-\left[Q\left(x_{u^{\prime}}\left(T, x^{\prime}\right)\right)-Q\left(x^{\prime}\right)-\epsilon\right] .
\end{aligned}
$$

Noting that $Q(x)$ is a Lipschitz function, $f(x)$ and $G(x)$ are real analytic, and the state space is bounded, we deduce that

$$
\begin{aligned}
\left|V\left(t, x^{\prime}\right)-V\left(t, x^{\prime \prime}\right)\right| \leq & \left|Q\left(x^{\prime}\right)-Q\left(x^{\prime \prime}\right)\right|+\left|Q\left(x_{u^{\prime}}\left(T, x^{\prime}\right)\right)-Q\left(x_{u^{\prime}}\left(T, x^{\prime \prime}\right)\right)\right| \\
& +\left|Q\left(x_{u^{\prime \prime}}\left(T, x^{\prime}\right)\right)-Q\left(x_{u^{\prime \prime}}\left(T, x^{\prime \prime}\right)\right)\right|+\epsilon \\
\leq & C\left|x^{\prime}-x^{\prime \prime}\right|+\epsilon
\end{aligned}
$$

by means of Gronwall inequality [2, page 829]. Since $\epsilon>0$ is arbitrary, we see that $V(t, x)$ is a Lipschitz function with respect to $x$. 
4. The algorithm for computing an optimal endpoint and an optimal control. The discussion in Section 3 suggests the following algorithm which includes the process of pre-estimation and correction.

Algorithm 4.1. (i) Take the initial value $x_{0}=\tilde{x}_{0}$.

(ii) (Pre-estimation). For $i=0,1,2, \ldots, L-1$, after having got $\tilde{x}_{i}$, we solve

$$
\begin{aligned}
& V\left[t_{i+1}, \tilde{x}_{i}+\tau f\left(\tilde{x}_{i}\right)+\sum_{k=1}^{m} \tilde{S}_{k}(i) g_{k}\left(\tilde{x}_{i}\right)\right] \\
& \quad=V\left(0, x_{0}\right)+Q\left(x_{0}\right)-Q\left[\tilde{x}_{i}+\tau f\left(\tilde{x}_{i}\right)+\sum_{k=1}^{m} \tilde{S}_{k}(i) g_{k}\left(\tilde{x}_{i}\right)\right]
\end{aligned}
$$

for $\tilde{S}_{k}(i), k=1,2, \ldots, m$, satisfying $\left|\tilde{S}_{k}(i)\right| \leq T \sup _{u \in U}\|u\| / L, k=1,2, \ldots$, $m$, to get

$$
x_{i+1}=\tilde{x}_{i}+\tau f\left(\tilde{x}_{i}\right)+\sum_{k=1}^{m} \tilde{S}_{k}(i) g_{k}\left(\tilde{x}_{i}\right) \text {. }
$$

(iii) (Correction). For $i=1,2, \ldots, L-1$, we take an admissible control $\tilde{u}_{i}(\cdot)$ on each $\left[t_{i}, t_{i+1}\right]$ such that

$$
\tilde{S}_{k}(i)=\int_{t_{i}}^{t_{i+1}} \tilde{u}_{i}^{(k)}(s) d s
$$

Define $\tilde{x}_{i+1}$ to be the state point to which the control $\tilde{u}_{i}(\cdot)$ steers system (1.1) from $\tilde{x}_{i}$ in the time interval $\left[t_{i}, t_{i+1}\right]$.

REMARK 4.2. In the process of this algorithm, we see that for $i=0,1, \ldots, L-1$, $\tilde{x}_{i+1}$ is determined by $\tilde{x}_{i}$ and $x_{i+1}$. Under this algorithm, we have, by (3.22),

$$
0=V\left(T, \tilde{x}_{L}\right)=V\left(0, x_{0}\right)+Q\left(x_{0}\right)-Q\left(\tilde{x}_{L}\right)+O\left(\tau^{2}\right)
$$

and produce the admissible control $\tilde{u}(\cdot)$ (which equals $\tilde{u}_{i}(\cdot)$ on each time interval $\left.\left[t_{i}, t_{i+1}\right], i=0,1,2, \ldots, L-1\right)$ which steers (1.1) from $x_{0}$ to $x_{\tilde{u}}\left(T, x_{0}\right)=\tilde{x}_{L}$.

In the following, we indicate that carrying out Algorithm 4.1, we can compute to get approximation to an optimal endpoint and an optimal control in general.

THEOREM 4.3. Suppose that there is an optimal control $\hat{u}(\cdot)$ for problem (1.1), (1.2), and (1.3). Denote by $\hat{x}(T)$ the endpoint of (1.1) with respect to $\hat{u}(\cdot)$, that is,

$$
Q(\hat{x}(T))=\min _{u \in U}\left\{Q\left(x_{u}(T)\right)\right\}
$$


Meanwhile, for each positive integer $L$, with $\tau=T / L$, let $\tilde{u}(\cdot)$ and $\tilde{x}_{L}$ be obtained by Algorithm 4.1. Then there is a positive real $C$ which only depends on the state space $X$ (which is assumed to be bounded) and $f(x), G(x)$ such that, for every large positive real $L(\tau=T / L)$,

$$
\left|Q\left(\tilde{x}_{L}\right)-Q(\hat{x}(T))\right| \leq C \tau^{2}
$$

Proof. By (3.20) and (3.21), we have

$$
\begin{gathered}
x_{L}-\tilde{x}_{L}=O\left(\tau^{2}\right), \\
Q\left(x_{L}\right)-Q\left(\tilde{x}_{L}\right)=O\left(\tau^{2}\right) .
\end{gathered}
$$

Noting (see Algorithm 4.1(ii))

$$
V\left(T, x_{L}\right)=V\left(0, x_{0}\right)+Q\left(x_{0}\right)-Q\left(x_{L}\right),
$$

we have, by (4.7) and (4.8),

$$
V\left(T, x_{L}\right)=V\left(0, x_{0}\right)+Q\left(x_{0}\right)-Q\left(\tilde{x}_{L}\right)+O\left(\tau^{2}\right)
$$

By noting that, for each $x \in X, V(T, x)=0$, we deduce, from (4.10), that

$$
V\left(0, x_{0}\right)=Q\left(\tilde{x}_{L}\right)-Q\left(x_{0}\right)+O\left(\tau^{2}\right) \text {. }
$$

Since $\hat{x}$ is the optimal endpoint, by the definition of $V\left(0, x_{0}\right)$ (see (3.1)), we have

$$
V\left(0, x_{0}\right)=Q(\hat{x})-Q\left(x_{0}\right)
$$

Combining (4.11) and (4.12), noting that the state space is assumed to be bounded, we conclude that there is a positive constant $C$ :

$$
\left|Q\left(\tilde{x}_{L}\right)-Q(\hat{x}(T))\right| \leq C T^{2} .
$$

At the end of this section, we would state that in some cases by Algorithm 4.1 one can compute an exact optimal control. For example, if we consider the simple linear system $\dot{x}=u$ in $R^{1}$, the algorithm will be as follows.

(i) Take the initial value $x_{0}=\tilde{x}_{0}$.

(ii) For $i=0,1,2, \ldots, L-1$, while having got $\tilde{x}_{i}$, we solve

$$
V\left[t_{i+1}, \tilde{x}_{i}+S(i)\right]=V\left(0, x_{0}\right)+Q\left(x_{0}\right)-Q\left[\tilde{x}_{i}+S(i)\right]
$$


for $S(i)$, satisfying $|S(i)| \leq T \sup _{u \in U}\|u\| / L$, to get

$$
\tilde{x}_{i+1}=\tilde{x}_{i}+S(i)
$$

(iii) For $i=1,2, \ldots, L-1$, take an admissible control $\tilde{u}_{i}(\cdot)$ on each $\left[t_{i}, t_{i+1}\right]$ such that

$$
S(i)=\int_{t_{i}}^{t_{i+1}} \tilde{u}_{i}^{(k)}(s) d s
$$

It is easy to see that $\tilde{x}_{i+1}$ is just the state point to which the control $\tilde{u}_{i}(\cdot)$ steers the system $\dot{x}=u$ from $\tilde{x}_{i}$ in the time interval $\left[t_{i}, t_{i+1}\right]$. Under this algorithm, we have, noting $V(T, x)=0$ for each $x \in X$,

$$
0=V\left(T, \tilde{x}_{L}\right)=V\left(0, x_{0}\right)+Q\left(x_{0}\right)-Q\left(\tilde{x}_{L}\right)
$$

and produce the admissible control $\tilde{u}(\cdot)$ which equals $\tilde{u}_{i}(\cdot)$ on each time interval $\left[t_{i}, t_{i+1}\right], i=0,1,2, \ldots, L-1$, and steers (1.1) from $x_{0}$ to $x_{\tilde{u}}(T)=\tilde{x}_{L}$. Together with (4.17), noting the definition of $V\left(0, x_{0}\right)$, we see that $\tilde{u}(\cdot)$ is the optimal control.

5. An example demonstrating the results of Section 4. We would demonstrate the above process by the following simple example.

EXAMPLE 5.1. Consider the following system in $R^{1}$ :

$$
\dot{x}=x u, \quad x(0)=1, \quad t \in[0,1], \quad u \in[-1,1] .
$$

Let $Q(x)=x$. We pose the optimal control problem

$$
\min _{u \in[-1,1]} Q\left(x_{u}(1)\right)
$$

Since $u(\cdot)$ is integrable and $|u| \leq 1$, we have

$$
V(t, x)=\inf _{u}\left\{\left[x e^{\int_{t}^{1} u(s) d s}\right]-x\right\}= \begin{cases}x\left(e^{t-1}-1\right), & \text { for } x \geq 0 \\ x\left(e^{1-t}-1\right), & \text { for } x<0 .\end{cases}
$$

Now the difference equations for $i \in\{0,1,2, \ldots, L-1\}(L>2)$ are as follows. First, corresponding to (3.9), we have

$$
x_{i+1}=x_{i}+S(i) x_{i}
$$


By noting that $x_{0}=1>0$ and $|S(i)|<1$ (for $L>2$ ), we see that $x_{i}>0, i=$ $0,1, \ldots, L$. Another equation (corresponding to (3.11)) for each $i$ is

$$
x_{i+1}\left(e^{t_{i+1}-1}-1\right)=e^{-1}-x_{i+1},
$$

that is,

$$
x_{i+1}=e^{-t_{i+1}} .
$$

By noting that $t_{i}=i / L, i=0,1,2, \ldots, L-1, L$, we see that, by (5.4) and (5.6),

$$
S(i)=e^{-1 / L}-1
$$

and we can choose $\tilde{u}_{i}(\cdot)=L\left(e^{-1 / L}-1\right)$ on $\left[t_{i}, t_{i+1}\right]$ by the following integral equality:

$$
\int_{t_{i}}^{t_{i+1}} u(s) d s=e^{-1 / L}-1
$$

which steers $x_{i}$ to

$$
\tilde{x}_{i+1}=x_{i} e^{\int_{t_{i}}^{t_{i+1}} \tilde{u}_{i}(s) d s}=x_{i} e^{e^{-1 / L-1}}
$$

By this process, we have, by (5.6),

$$
\begin{aligned}
x_{i+1}-\tilde{x}_{i+1} & =e^{-(i+1) / L}-e^{-i / L} e^{e^{-1 / L}-1} \\
& =e^{-(i+1) / L}\left[1-e^{\left\{1 / L+e^{-1 / L}-1\right\}}\right]=O\left(\frac{1}{L^{2}}\right) .
\end{aligned}
$$

Finally, from (5.6), (5.7), (5.8), and (5.9), we see that

$$
\tilde{x}_{L}=e^{-(L-1) / L} e^{e^{-1 / L}-1}=e^{-1} e^{1 / L} e^{e^{-1 / L}-1} \longrightarrow e^{-1} \quad(L \longrightarrow \infty) .
$$

It is easy to see that the optimal control for this problem is $\hat{u}(\cdot) \equiv-1$ and the optimal endpoint is $\hat{x}(1)=e^{-1}$. Note that Algorithm 4.1 suggests that the approximation of the optimal control for this problem is $\tilde{u}(\cdot) \equiv L\left(e^{-1 / L}-1\right)$ which tends to -1 as $L \rightarrow \infty$, by (5.11).

\section{An application in singular linear quadratic optimal control problems.} We would like to present an application of the approximation approach in Sections 2 and 3 in singular linear quadratic optimal control problems by the following example. We aim to find a discrete optimal feedback control. 
EXAMPLE 6.1. Consider the following linear control system in $R^{1}$ :

$$
\dot{x}=x+u, \quad x(0)=\frac{1}{3}, \quad t \in[0,1], \quad u \in U=[-1,1],
$$

where we consider the admissible control $u(\cdot)$ to be piecewise continuous. We define the quadratic cost functional

$$
J(u)=\int_{0}^{1}\left(x^{2}+x u\right) d t
$$

and pose the optimal control problem: find an admissible control $\hat{u}(\cdot)$ such that

$$
J(\hat{u})=\min _{u \in U} J(u)
$$

We define the value function for problem (6.1), (6.2), and (6.3):

$$
V(t, x)=\inf _{u \in U} \int_{t}^{1}\left(x_{u}^{2}+x_{u} u\right) d s
$$

where $x_{u}$ is the solution of $\dot{x}=x+u, x(t)=x$, and $s \in[t, 1]$.

Next we transfer problem (6.1), (6.2), and (6.3) into the Mayer problem which is equivalent to the original problem. Consider the system

$$
\dot{x}=x+u, \quad \dot{y}=x^{2}+x u, \quad x(0)=\frac{1}{3}, \quad y(0)=0, \quad t \in[0,1],
$$

and find the admissible control $\hat{u}(\cdot)$ such that

$$
y_{\hat{u}}(1)=\min _{u \in U} y_{u}(1)
$$

If, according to (3.1), we define the value function of problem (6.5) and (6.6):

$$
\tilde{V}(t, x, y)=\min _{u \in U}\left(y_{u}(t, 1)-y\right)
$$

where $y_{u}(t, 1)$ is the solution of $\dot{x}=x+u, \dot{y}=x^{2}+x u, x(t)=x, y(t)=y$, and $s \in[t, 1]$, we see that

$$
V(t, x)=\tilde{V}(t, x, y)
$$

To carry out Algorithm 4.1 for this example, for given positive integer $L$, we divide $[0,1]$ into $L$ equal parts: $[i / L,(i+1) / L], i=0,1,2, \ldots, L-1$. Denote $t_{i}=i / L, i=0,1,2, \ldots, L-1$. The iterative process is as follows:

(i) take the initial values

$$
\tilde{x}_{0}=\frac{1}{3}, \quad \tilde{y}_{0}=0 ;
$$


TABLE 6.1

\begin{tabular}{r|cc}
\hline$L$ & Control $(u)$ & Trace $(\tilde{x})$ \\
\hline 0 & -0.333333333 & 0.333333333 \\
1 & -0.333333333 & 0.333333333 \\
2 & -0.333333333 & 0.333333333 \\
3 & -0.333333333 & 0.333333333 \\
4 & -0.333333333 & 0.333333333 \\
5 & -0.333333333 & 0.333333333 \\
6 & -0.333333333 & 0.333333333 \\
7 & -0.333333333 & 0.333333333 \\
8 & -0.333333333 & 0.333333333 \\
9 & -0.333333333 & 0.333333333 \\
10 & -0.333333333 & 0.333333333 \\
11 & -0.347917818 & 0.333333333 \\
12 & -0.622540238 & 0.332585571 \\
13 & -0.877211794 & 0.317719277 \\
14 & -0.934028646 & 0.289033482 \\
15 & -0.929048395 & 0.255963873 \\
16 & -0.912656792 & 0.221454092 \\
17 & -0.888671881 & 0.186015371 \\
18 & -0.863446877 & 0.149989402 \\
18 & -0.84136498 & 0.113409655 \\
20 & - & 0.076086587 \\
\hline
\end{tabular}

(ii) if $\tilde{x}_{i}$ and $\tilde{y}_{i}$ are obtained, solve

$$
V\left(t_{i+1}, \tilde{x}_{i}+\frac{1}{L} \tilde{x}_{i}+S(i)\right)=V\left(0, \frac{1}{3}\right)-\tilde{y}_{i}-\frac{1}{L} \tilde{x}_{i}^{2}-S(i) \tilde{x}_{i}
$$

for $S(i)$ satisfying $|S(i)| \leq 1 / L$. Here we apply optimization method to compute $V(t, x)$ and approximate $S(i)$ by an iterative process. Then compute

$$
\tilde{y}_{i+1}=\tilde{y}_{i}+\frac{1}{L} \tilde{x}_{i}^{2}+S(i) \tilde{x}_{i}
$$

(iii) define $\hat{u}_{i}=L S(i)$ and compute

$$
\tilde{x}_{i+1}=e^{1 / L}\left[\tilde{x}_{i}+L S(i)\left(e^{-t_{i}}-e^{-t_{i+1}}\right)\right],
$$

then, go to (ii).

Carrying out the iterative process via Matlab, we list the data of $\tilde{x}_{i}$ and $u_{i}$, $i=1,2, \ldots, L$, for $L=20$ in Table 6.1 . 


\section{REFERENCES}

[1] C.-S. Huang, S. Wang, and K. L. Teo, Solving Hamilton-Jacobi-Bellman equations by a modified method of characteristics, Nonlinear Anal., Ser. A: Theory Methods 40 (2000), no. 1-8, 279-293.

[2] F. Lamnabhi-Lagarrigue and G. Stefani, Singular optimal control problems: on the necessary conditions of optimality, SIAM J. Control Optim. 28 (1990), no. 4, 823-840.

[3] H. J. Sussmann, A sufficient condition for local controllability, SIAM J. Control Optim. 16 (1978), no. 5, 790-802.

[4] L Lie brackets and local controllability: a sufficient condition for scalar-input systems, SIAM J. Control Optim. 21 (1983), no. 5, 686-713.

[5] _ A general theorem on local controllability, SIAM J. Control Optim. 25 (1987), no. 1, 158-194.

[6] H. J. Sussmann and V. Jurdjevic, Controllability of nonlinear systems, J. Differential Equations 12 (1972), 95-116.

Jinghao Zhu: Department of Applied Mathematics, School of Science, Tongji University, Shanghai 200092, China

E-mail address: jinghaok@on1ine.sh.cn

Zhiqiang Zou: Department of Applied Mathematics, School of Science, Tongji University, Shanghai 200092, China

E-mail address: zqzou@sohu.com 


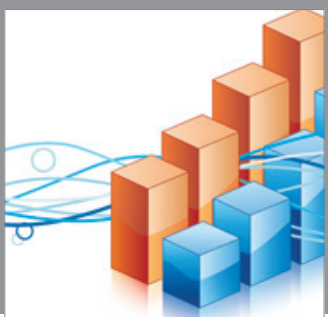

Advances in

Operations Research

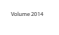

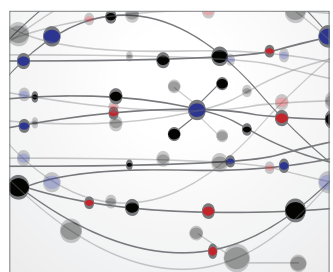

\section{The Scientific} World Journal
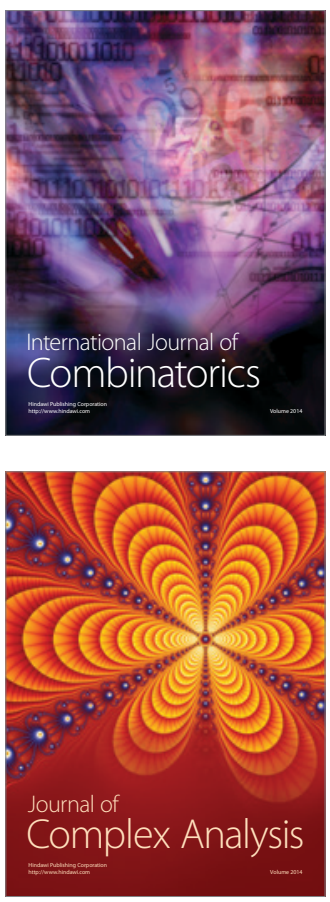

International Journal of

Mathematics and

Mathematical

Sciences
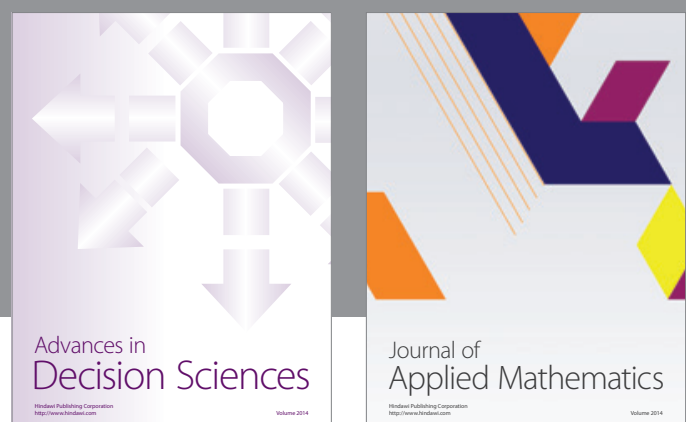

Journal of

Applied Mathematics
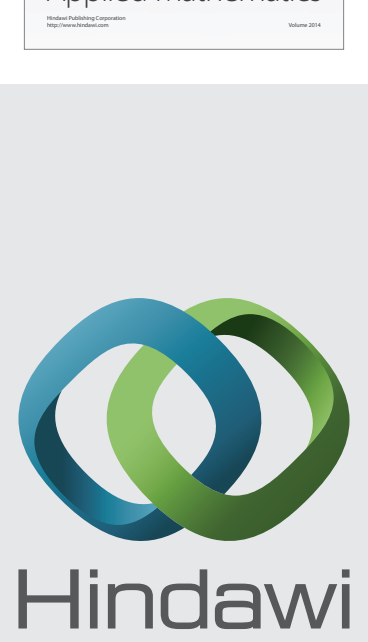

Submit your manuscripts at http://www.hindawi.com
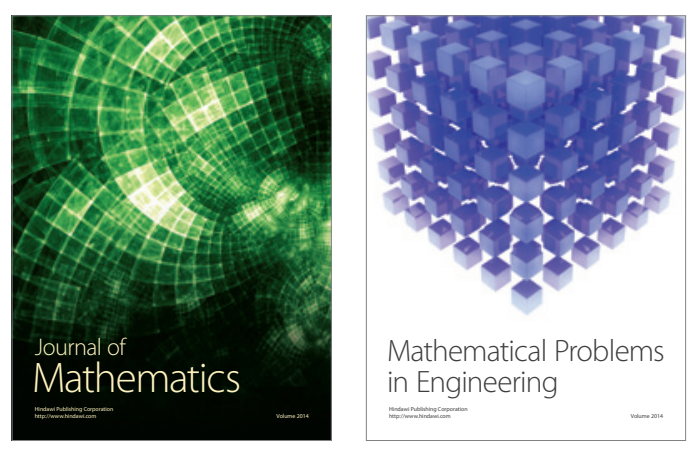

Mathematical Problems in Engineering
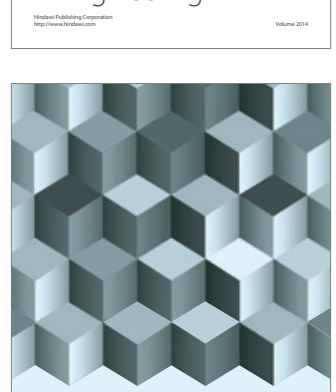

Journal of

Function Spaces
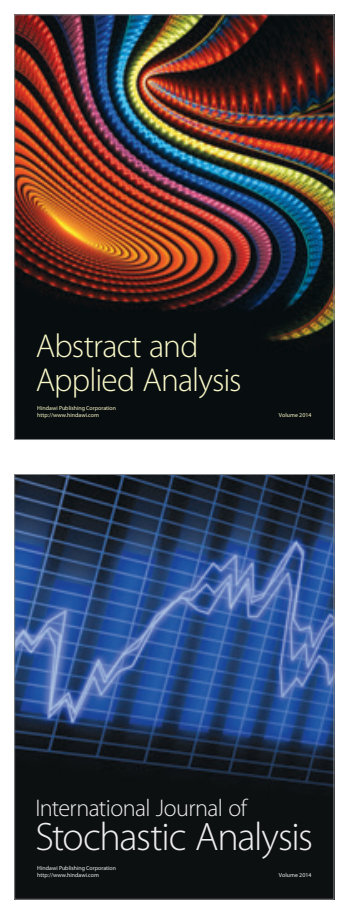

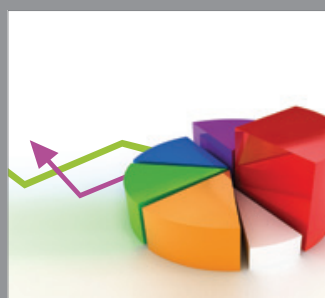

ournal of

Probability and Statistics

Promensencen
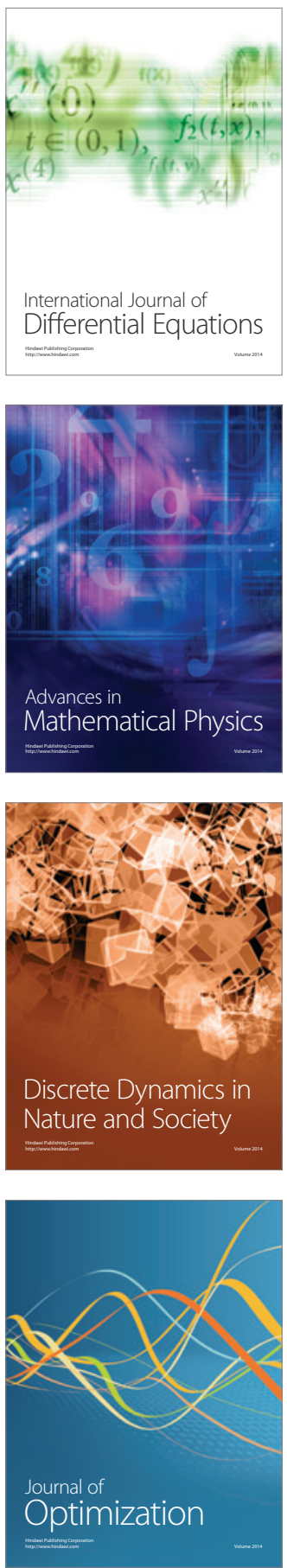